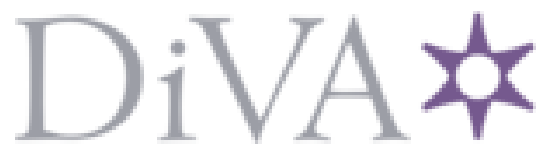

http://www.diva-portal.org

This is the published version of a paper presented at 1oth IEEE/ACM International Conference on Utility and Cloud Computing, Austin, Texas, USA, December 5-8, 2017.

Citation for the original published paper:

Ibidunmoye, O., Moghadam, M H., Lakew, E B., Elmroth, E. (2017)

Adaptive Service Performance Control using Cooperative Fuzzy Reinforcement

Learning in Virtualized Environments

In: UCC'17 Proceedings of the1oth International Conference on Utility and Cloud

Computing (pp. 19-28). IEEE/ACM

https://doi.org/10.1145/3147213.3147225

N.B. When citing this work, cite the original published paper.

Permission to make digital or hard copies of all or part of this work for personal or classroom use is granted without fee provided that copies are not made or distributed for profit or commercial advantage and that copies bear this notice and the full citation on the first page. Copyrights for components of this work owned by others than ACM must be honored. Abstracting with credit is permitted. To copy otherwise, or republish, to post on servers or to redistribute to lists, requires prior specific permission and/or a fee. Request permissions from permissions@acm.org.

Permanent link to this version:

http://urn.kb.se/resolve?urn=urn:nbn:se:umu:diva-142032 


\section{Adaptive Service Performance Control using Cooperative Fuzzy Reinforcement Learning in Virtualized Environments}

\author{
Olumuyiwa Ibidunmoye \\ Umeå University \\ Department of Computing Science \\ SE-901 87 \\ Umeå, Sweden \\ muyi@cs.umu.se
}

\author{
Mahshid Helali Moghadam \\ University of Kashan \\ Department of Computer Engineering \\ Kashan, Iran \\ mhelali@grad.kashanu.ac.ir
}

\author{
Ewnetu Bayuh Lakew, \\ Erik Elmroth \\ Umeå University \\ Department of Computing Science \\ SE-901 87 \\ Umeå, Sweden \\ \{ewnetu,elmroth\}@cs.umu.se
}

\begin{abstract}
Designing efficient control mechanisms to meet strict performance requirements with respect to changing workload demands without sacrificing resource efficiency remains a challenge in cloud infrastructures. A popular approach is fine-grained resource provisioning via auto-scaling mechanisms that rely on either threshold-based adaptation rules or sophisticated queuing/control-theoretic models. While it is difficult at design time to specify optimal threshold rules, it is even more challenging inferring precise performance models for the multitude of services. Recently, reinforcement learning have been applied to address this challenge. However, such approaches require many learning trials to stabilize at the beginning and when operational conditions vary thereby limiting their application under dynamic workloads. To this end, we extend the standard reinforcement learning approach in two ways: a) we formulate the system state as a fuzzy space and b) exploit a set of cooperative agents to explore multiple fuzzy states in parallel to speed up learning. Through multiple experiments on a real virtualized testbed, we demonstrate that our approach converges quickly, meets performance targets at high efficiency without explicit service models.
\end{abstract}

\section{CCS CONCEPTS}

- Computer systems organization $\rightarrow$ Cloud computing; $\bullet$ Networks $\rightarrow$ Network performance evaluation; • Computing methodologies $\rightarrow$ Machine learning;

\section{KEYWORDS}

Performance control; Resource allocation; Quality of service; Reinforcement learning; Autoscaling; Autonomic computing.

\section{INTRODUCTION}

One of the main attractiveness of cloud computing is the ability to provision computing resources (e.g. compute, memory, storage, etc.) on demand on a pay-as-you-go basis. Thanks to advancements

Permission to make digital or hard copies of all or part of this work for personal or classroom use is granted without fee provided that copies are not made or distributed for profit or commercial advantage and that copies bear this notice and the full citation on the first page. Copyrights for components of this work owned by others than ACM must be honored. Abstracting with credit is permitted. To copy otherwise, or republish, to post on servers or to redistribute to lists, requires prior specific permission and/or a fee. Request permissions from permissions@acm.org.

UCC'17, December 5-8, 2017, Austin, Texas, USA

(C) 2017 Association for Computing Machinery.

ACM ISBN 978-1-4503-5149-2/17/12 ..\$15.00

https://doi.org/10.1145/3147213.3147225 in virtualization technologies, cloud infrastructures enable applications to achieve rapid elasticity by dynamically acquiring and releasing resources on the fly according to changing workload levels. However, determining the right amount of resource capacity to increase or decrease automatically remains a challenge given the dynamic nature of workloads, plurality of services and quality of service $(\mathrm{QoS})$ requirements.

Autoscaling $[17,19]$ is a technique that have been employed to address the aforementioned challenge on different fronts. It involves automatically adjusting the amount of resources available to an application in response to changing workload demand in order to meet QoS requirements (e.g. performance and resource efficiency). Autoscaling techniques typically scale applications in two wayshorizontally and vertically. Vertical scaling modifies the amount of resources allocated to a service's virtual machine (VM) such as CPU cores or memory. Horizontal scaling adjusts the number of VMs available to a service often with a higher configuration latency. The later is more widely available in commercial clouds (e.g. Amazon, Google, Windows Azure, Rackspace, etc.) as it requires no extra support from the hypervisor unlike the former which may require rebooting and has to be supported by both the hypervisor and guest operating system's kernel [15]. While only a few providers such as CenturiLink $^{1}$ and ProfitBricks ${ }^{2}$ offer some form of vertical scaling, most modern hypervisors such as Xen, KVM and VMware provides built in support such as CPU hot-plugging, capping and memory ballooning [19]. We focus on vertical scaling in this paper since it is less explored than the horizontal case. In addition, since fractional resource capacity (e.g. CPU core) can be allocated with configuration latency of less than $0.5 \mathrm{~s}$ [21], it can compliment horizontal scaling towards truly actualizing the pay-as-use pricing model.

Autoscaling solutions rely on a variety of strategies for decision making. The most basic strategy is based on a set of predefined IF..THEN rules consisting of logical conditions (e.g. CPU\%>70) and corresponding actions (e.g. adding 1 more CPU core) $[4,9,10]$. In order to cope with the limitation of rule-based techniques under dynamic workloads, sophisticated approaches build analytical models of execution environments using techniques from a variety of mathematical disciplines $[1,6-8,11,15,21,23]$. However, accurate estimation of scaling models often require detail knowledge of service and workload characteristics thereby limiting their applicability to handle heterogeneous service mix. Recently, reinforcement

\footnotetext{
${ }^{1}$ https://www.ctl.io/autoscale/\#VerticalAutoscale

${ }^{2}$ https://www.profitbricks.com/help/Live_Vertical_Scaling
} 
learning (RL) has been applied to achieve a more generic approach to the autoscaling problem without precise model of service workloads and its execution context $[5,12,13,16]$. However, the bad initial performance-requiring many learning trials to stabilize at the beginning and when conditions change required by such approaches limits their application in real-time deployments [17].

In this paper, we present the design and evaluation of an adaptive mechanisms for meeting QoS requirements (such as performance SLA and resource efficiency) using cooperative fuzzy reinforcement learning (CoFReL). Specifically, CoFReL extends the standard RL (Q-learning) approach in two ways to speed up learning: a) we formulate the system state as a fuzzy space and b) exploit a set of cooperative agents to explore multiple fuzzy states in parallel. The hypothesis is that a set of cooperating agents sharing a collective experience explore the state space faster than a single agent. We demonstrate the efficacy of our approach through multiple experiments on a real virtualized testbed. In particular, our technique converges, on average, within the first 11 learning cycles with an overall average convergence rate of 6.7 learning cycles. In addition to meeting performance SLA even for response time targets as low as $0.5 \mathrm{~s}$, it consistently achieve a resource efficiency of at least $96 \%$ across workloads.

The rest of the paper is organized as follows; Section 2 discusses background and motivation while Section 3 summarizes the proposed approach while technical details of its main components discussed in Section 4 and Section 5. Experimental evaluation and results are presented in Section 6. The paper concludes with a review of Related Work in Section 7 and Conclusion in Section 8.

\section{MOTIVATION AND BACKGROUND}

\subsection{Motivation}

Though virtualization technologies allows cloud service providers to scale services and optimize server utilization in a cost-effective way, balancing the trade-off between efficient utilization of allocated resources and meeting performance service-level agreements (SLA) remains a major objective. On one hand, allocating resources in excess of actual demand-overprovisioning-results in wastage due to under-utilization and extra monetary cost, while on the other hand, insufficient resource allocations-underprovisioning-causes performance degradation and SLA violations.

Model-driven autoscaling solutions targeting this trade-off belong to one of two groups: a) white-box techniques based on queuing models [7, 23], fuzzy control [11], b) black-box techniques based on control theory $[1,6,15]$,time-series and predictive analysis [8]. While the white-box techniques may demand precise system model based on knowledge of service composition, propagation of service requests and workload characteristics, the black-box may require different models for individual different QoS metrics (e.g. response time, throughput) and scenarios (e.g. average vs tail latency or scale-up vs scale-down).

Nevertheless, major barriers to achieving adaptive performance control in cloud environments include a) the complexity and heterogeneity of infrastructures and services making it hard to craft precise models of the execution environment and $b$ ) the dynamism of workloads making workload predictions and consequent scaling decisions unreliable. These characteristics suggest the need for a more generic framework for performance control in heterogeneous environments which does not require precise model of services nor their workloads.

\subsection{Reinforcement Learning for Adaptive Performance Control}

Adaptive performance control is an interactive decision-making problem involving a controller which continuously monitors one or more QoS indicators (e.g. performance, utilization) of a controlled system and dynamically decreases or increases resource capacity to maintain the QoS at a desired target level. The controlled system in this paper is a virtualized application deployed in a VM hosted in an Infrastructure as a Service (IaaS) cloud.

Reinforcement Learning (RL) [22] is a mechanism that can be used for handling such interactive scenarios. This interaction involves an agent (the controller) which continually senses the state (e.g. latency, CPU usage) of the environment (system or application). The agent uses its experiences to select an action (e.g. add/remove $\mathrm{CPU}$ cores) to be applied to the environment which affects the state and in return receives a reinforcement signal in the form of a scalar reward (e.g. -1 for a bad action and +1 for a good action in a specific state). In order to meet a given learning goal (e.g. meeting latency targets), the agent follows a policy that takes actions in a way that maximizes the long-term cumulative reward. The agent autonomously learns this policy via a systematic trial-and-error scheme of selecting high-value actions (exploitation) and trying out a variety of actions to discover good ones (exploration). In general, it is supposed that the environment is non-deterministic, so that applying the same action to the environment on two occasions may lead to two different next states and/or reinforcement signal values. However, it is also supposed that the environment is stationary, so that the probabilities of states transitions or receiving specific values of reinforcement signal is fixed over the time.

To find the optimal policy, the agent must sequentially estimate the utility the long-term cumulative reward of taking each action in the each state. Q-learning [22] is an RL algorithm for learning such functions in an online manner. It is an off-policy technique as the value function is learned independently of the policy being followed by the agent. Once the optimal policy is learned, the agent will replay it while occasionally trying out unexplored actions.

\section{SYSTEM OVERVIEW}

In this section we briefly present an overview of our framework for adaptive performance control using a model-free technique which we call Cooperative Fuzzy Reinforcement Learning (CoFReL). As shown in Fig. 1, CoFReL consists of two main components namely:

1) The State Detector is responsible for observing the current state of the controlled system and computing associated rewards. It receives the values of the QoS metrics at time $t$; the Response Time, $R T_{t}$, and CPU utilization, $C P U_{t}$ of the controlled service via a sensor. ${ }^{3}$ The metrics are combined using fuzzy rules to classify the system into one or more fuzzy states. The reward, $R_{t}$, is derived using continuous function that quantifies the utility of the system in terms of compliance with given SLA target and

\footnotetext{
${ }^{3}$ Note that time-indexed variables will be written in the form $X_{t}$ rather than the standard functional form $X(t)$ throughout this paper.
} 


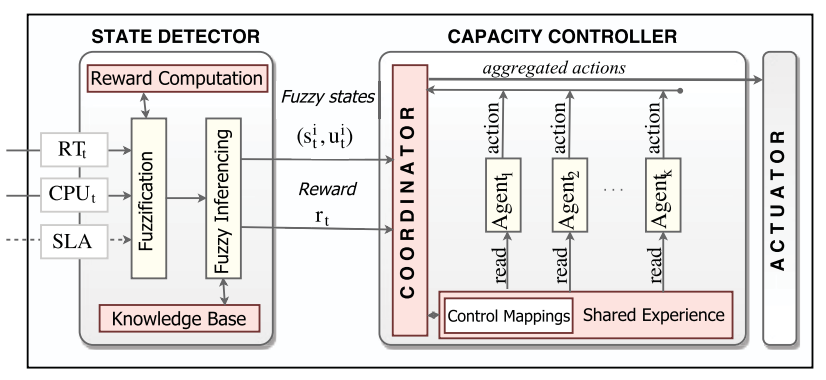

Figure 1: System Overview

achieved resource efficiency. In this paper, we consider performance SLA characterized by a target value, $\tau$, and a tolerance band, $t o l=\left[\tau^{-}, \tau^{+}\right]$where $\tau^{-}=\tau-\varepsilon, \tau^{+}=\tau+\varepsilon$, and $\varepsilon$ is a small tolerance value.

2) The Capacity Controller employs semi-supervised learning to autonomously adjust the amount of compute capacity available to the service VM in order to adapt to incoming workload. Inputs to the capacity controller includes the set of observed fuzzy states and the reward of the action taken in the previous step. The major building block of the controller is a coordinator which manages a set of cooperative learning agents to explore multiple fuzzy states in parallel. Based on the current states and the memory of shared experience, each agent recommends a scaling action (e.g. add or remove a fractional capacity). To make capacity actuation atomic as much as possible, the coordinator aggregates actions by taking the arithmetic sum of recommended actions each scaled by the degree of membership in the corresponding state. Finally, the coordinator updates the shared experience. The aggregated action is applied to the service VM using VM management API of the hypervisor. Capacity actuations are applied in an incremental fashion, so that total capacity after an action is an accumulation of past actuations. To improve control stability and speed up state-action exploration, the administrator can define control mappings which are high-level rules to guide the agents on what category of actions not to explore when in a specific state.

We discuss further the technical details of two components in sections 4 and 5 respectively.

\section{STATE DETECTION}

Sensing and recognizing states of the controlled system is a major task in a reinforcement learning problem. According to Fig. 2, the system state is represented as a two dimensional space of the two QoS indicators to be optimized namely performance (Response Time) and resource usage (CPU utilization). Whereas the former acts like a proxy for end-user performance, the later indirectly captures the service workload. Also, due to the imprecise nature of state boundaries the state formulation is such that the system can be in one or more state(s) at varying degrees. To address the uncertainty in the state space, we apply fuzzy membership functions and fuzzy rules [2], in Section 4.1, to characterize system state at any given time. In addition, the computation of rewards is performed externally from the controller, as part of state detection, so that the agents do not have control over how rewards are estimated thereby preventing overcompensation. In Section 4.2 we describe a continuous utility function for computing rewards.

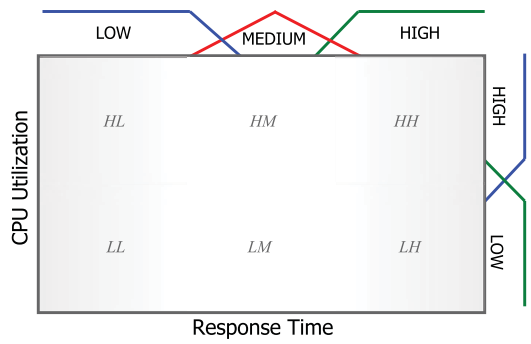

Figure 2: 2-dimensional fuzzy state representation.

\subsection{Fuzzy State Classification}

The standard state detection in RL problems typically assumes that states are mutually exclusive. For example, in Fig. 2, it would be supposed that the system can be in only a distinct state $\mathrm{HL}$, for example, when the CPU utilization is High and Response Time is Low. The challenge of such crisp representation includes knowing what is high enough to be a good threshold for each attribute's category, how to treat boundary values, as well as how to recognize vague expert concepts of the environment such as Very Low or Medium. Fuzzy logic facilitates the formalization of these kind of uncertainties using interpretable fuzzy rules based informal domain knowledge and simple fuzzy operators.

Fuzzy state classification [14] is a form of soft labelling operation in which an QoS measurement, $\left(R T_{t}, C P U_{t}\right)$, of the controlled system is assigned to one or more states with varying degrees using fuzzy sets and rules (soft labeling). While $C P U$ metric is bounded between $[0 \%, 100 \%]$, the $R T$ metric is not so bounded, so the domain of $R T$ is normalized to interval $[a, b]$ using an inverse tangent function as follows:

$$
R T_{t}=b \cdot \frac{2}{\pi} \tan ^{-1}\left(\frac{R T_{t}^{\prime}}{\tau}-a\right)
$$

where $R T_{t}^{\prime}$ is the measured response time at time $t, \tau$ is the target value (SLA), while $a$ and $b$ are the lower and upper response time limits set to 0 and 100 similar to the $C P U$ metric. The implication of this function is such that when $R T_{t}^{\prime}=\tau$, the normalized value is 50 and $R T_{t}^{\prime}>\tau$ are normalized to values towards 100 while $R T_{t}^{\prime}<\tau$ are normalized towards 0 . The normalization is necessary to ensure that the $2 \mathrm{D}$ state-space is bounded which simplifies reasoning and exploration of the state space.

Fuzzification involves defining category membership functions over the domain of each QoS metric [2]. A category membership function is identified by a linguistic term such as Low, or High mapped to a fuzzy set and the set of all membership functions of a given metric is an overlapping ranges of values over its domain. A fuzzy set $A$ in a continuous domain $X: \mathbb{R}$ is defined as $A=\left\{\left(x, \mu_{A}(x)\right) \mid x \in X\right\}$ and characterized by a membership function $\mu_{A}: x \rightarrow[0,1]$. For all $x \in X, \mu_{A}(x)$ describes the degree to which $x$ belongs in $A$. Though the membership function, $\mu_{A}(x)$, can take one of several shapes [2], we have used trapezoidal for 
Low and High categories and triangular for Medium as shown in Fig. 2. In our case, ranges of membership functions are estimated empirically and can be automatically updated to reflect prevailing workload behaviour.

Fuzzy Inferencing allows us to reason about all possible states the system can assume using fuzzy rules constructed from domain knowledge [2]. A rule, as shown in Eq.(2), is made up of two parts namely the antecedent-logical combination of the linguistic terms of one or more variables using appropriate fuzzy operators, and the consequent which determines the extent of being in the associate state.

$$
\begin{aligned}
& f_{1}: \text { IF CPU\% is Low AND RT is High } \\
& \text { rule antecedent } \\
& \text { THEN STATE is LH } \\
& \text { rule consequent }
\end{aligned}
$$

The output variable of a rule is realised using a singleton membership function so that the output of each rule is the support for being in the associated state. Given an input vector, $\vec{x}$, the degree of activating rule $f_{i}$ or support for the associated state, is derived as $\tau(\vec{x})=\prod_{j=1}^{n} \mu_{A_{j}}\left(x_{j}\right)$ where $n$ is the number of linguistic terms in the rule. In our case, we have a total of six rules in total, so the output of the inference engine is a set of remaining state-degree pairs, $\left\{s_{t}^{i}, \mu_{t}^{i}\right\}$, after filtering those pairs where $\mu_{t}^{i}=0$.

\subsection{Reward Computation}

To compute the reward signal, we derive a utility of the system as a weighted combination of performance and efficiency as follows:

$$
U_{t}=\omega H_{t}+(1-\omega) E_{t}
$$

where $H_{t}$ is the degree of compliance with performance SLA and $E_{t}$ is the resource efficiency, and $\omega, 0 \leq \omega \leq 1$, is a multiplier to allow administrator prioritize either performance or efficiency. The SLA compliance, $H_{t}$, is estimated using a triangular function:

$$
H_{t}= \begin{cases}0, & R T_{t} \leq \tau^{-} \\ \frac{R T_{t}-\tau^{-}}{\tau-\tau^{-}}, & \tau^{-}<R T_{t} \leq \tau \\ \frac{\tau^{+}-R T_{t}}{\tau^{+}-\tau}, & \tau<R T_{t} \leq \tau^{+} \\ 0, & R T_{t} \geq \tau^{+}\end{cases}
$$

while resource efficiency, $E_{t}$, is the ratio between current $\mathrm{CPU}$ utilization and maximum utilization value possible, that is, $E_{t}=$ $C P U_{t} / C P U_{\text {max }}$. Note that $\tau^{-}$and $\tau^{+}$are the lower and upper limit of the tolerance region respectively. Finally, the reward value is computed as an inverse function of the hyperbolic tangent of the utility function:

$$
r_{t}=\frac{1}{1-\tanh \left(U_{t}\right)}
$$

A major property of Eq.(4) is that it ensures the continuity and proportionality of reward values, so that the lower the utility the lower the reward and vice versa. Also, the hyperbolic tangent acts like an activation function with finite output in the range $(-1,1)$. Since the hyperbolic tangent of boundary utility values 0 and 1 are defined, the reward function is stable with defined output.

The set of state-degree pairs, $\left\{s_{t}^{i}, \mu_{t}^{i}\right\}$, and corresponding scalar reward, $r_{t}$, form the input to the controller introduced in the next section.

\section{RESOURCE CONTROL USING COOPERATIVE FUZZY Q-LEARNING}

In this section, we describe the detail of the cooperative fuzzy $\mathrm{Q}-$ learning based capacity controller, introduced in Section 3.

The goal of the controller is to automatically learn auto-scaling policies to drive the system to desired state via incremental capacity adjustments. The learning task involves the coordination of $k$ parallel reinforcement learning agents each exploring the state-action space of a given state over all possible actions in that state. The controller interacts with the environment at discrete time steps, $t=0,1,2,3, \ldots$, . At each time $t$, the controller receives a set of state-degree pairs, $\left\{s_{t}^{i}, \mu_{t}^{i}\right\}_{i=1}^{k}$ from the state detector, and $s_{t}^{i} \in S$ is the $i$ th fuzzy state and $\mu_{t}^{i}$ is the extent of the system in that state. The controller then activates the associated agent for each fuzzy state $s_{t}^{i}$. Based on past experience maintained in a lookup table, the $q$-table, of state-action values, each agent recommends an action $a_{t}^{i} \in A\left(s_{t}^{i}\right)$ where $A\left(s_{t}^{i}\right)$ is the set of actions available in state $s_{t}^{i}$ and $A$ is the set of all possible actions. Actions involve increasing (scale-up) or decreasing (scale-down) the cumulative CPU capacity by a fractional factor in the set $\{0,2 / 5,3 / 5,4 / 5,1,3 / 2,2\}$.

To make actuations atomic, the coordinator aggregates recommendations by taking the arithmetic sum of recommended actions each scaled by the associated degree $\mu_{i}(t)$ :

$$
a_{t}=\sum_{i=1}^{k} \mu_{t}^{i} \cdot a_{t}^{i}
$$

The coordinator sends the aggregated action $a_{t}$ to the actuator which adjusts cumulative capacity of the system accordingly. After one time step, $t+1$, the agent receives a numerical signal $r_{t+1} \in \mathbb{R}$, the immediate reward, as well as a new set of fuzzy state-degree pairs $\left\{s_{t}^{i}, \mu_{t}^{i}\right\}_{i=1}^{\ell}$ and the procedure is repeated.

The learning goal is to find a policy $\pi: S \rightarrow A$ mapping every state, $s$, with the best action, $a$, which maximizes the expected discounted rewards over the future as follows [22]:

$$
R_{t}=r_{t+1}+\gamma r_{t+2}+\gamma^{2} r_{t+3}+\cdots=\sum_{k=0}^{\infty} \gamma^{k} r_{t+k+1}
$$

where $\gamma, 0 \leq \gamma \leq 1$, is a discount factor which determines the importance given to future rewards compared to immediate rewards as well as to avoid infinite rewards. Furthermore, the policy $\pi(s, a)$, the probability of taking action $a$ in state $s$, is associated with an expected utility value $Q^{\pi}(s, a)$ defined as [22]:

$$
Q^{\pi}(s, a)=E\left\{\sum_{k=0}^{\infty} \gamma^{k} r_{t+k+1} \mid s_{t}=s, a_{t}=a\right\}
$$

Each agent, at each control interval, relies on $Q^{\pi}(s, a)$ to make action recommendations, which captures the desirability of taking action $a$ while in state $s$ under the policy $\pi$.

Due to the infinite nature of Eq. 7, the quantity $Q^{\pi}(s, a)$ is typically evaluated in an online manner via temporal differencing. This involves maintaining an in-memory lookup table, the $q$-table, to keep track of the expected value of each state for all possible actions, and applying incremental updates to each state-action entry.

Given the fuzzy nature of our scenario, the update is based on two heuristics a) that the $q$-value of the state-action associated with each 
agent is updated individually according to their contribution to the aggregate action, and $\mathrm{b}$ ) that the cooperation between the $k$ agents at time $t$ moved the system from states $\left\{s_{t}^{i}\right\}_{i=1}^{k}$ to $\left\{s_{t}^{i}\right\}_{i=1}^{\ell}$. Hence, the reward $r_{t+1}$ obtained as a result of having taken aggregated action $a_{t}$ in the previous time step should be shared among the agents. The one-step cooperative fuzzy Q-learning update rule for each agent is defined as:

$$
\begin{gathered}
Q\left(s_{t}^{i}, a_{t}^{i}\right)=\left[(1-\alpha) Q\left(s_{t}^{i}, a_{t}^{i}\right)+\alpha\left(\mu_{t}^{i} \cdot r_{t+1}+\gamma V_{t+1}\right)\right] \mu_{t}^{i} \\
V_{t+1}=\max \left(\left\{\mu_{t}^{i} \cdot \max _{a^{\prime} \in A} Q\left(s_{t+1}^{i}, a^{\prime}\right)\right\}_{i=1}^{\ell}\right)
\end{gathered}
$$

Whereas the first term of Eq. 8 is the value of $s_{t}^{i}$ before action $a_{t}^{i}$ was taken, the second term is sum of shared reward and the best discounted return or utility value across all the resulting states (Eq. 9). Parameter $\alpha, 0 \leq \alpha \leq 1$, controls the rate of learning in terms of the impact of new utility value on the $q$-value.

Eventually, each agent will find the optimal policy $\pi^{*}$ which selects the action that maximizes the $q$-value of a given state $s$ :

$$
a(s)=\arg \max _{a^{\prime} \in A} Q\left(s, a^{\prime}\right)
$$

Adaptation. Following only Eq. 10, the agents will greedily select high-value actions, without trying out other actions that may yield even better value. The action selection procedure employs the $\epsilon$ greedy strategy to prevent this situation. With probability $1-\epsilon$, the best action according to Eq. 10 will be selected and with probability $\epsilon$ a random action will be chosen to explore a variety of actions thereby gaining more experience.

Improving learning performance. RL approaches generally suffer slow convergence rate due to unfeasibly long training time required to explore the state space using a single agent. This concern informed our fuzzy state detection and the use of cooperative agents to explore multiple fuzzy states in parallel. In addition, large action space may introduce undesirable fluctuations in controller behaviour which may introduce arbitrary spikes in end-user service performance. To attenuate this effect, we define control mappings based on high-level domain knowledge to guide the agents on what category of actions not to explore when in a specific state. For example, when in state LL of Fig. 2, agents can be discouraged from making high fractional increments since allocated resource is currently underutilized. The control mappings is implemented according to the following initialization:

$$
Q\left(s_{i}, a_{j}\right)=\left\{\begin{array}{cl}
-1, & \left(s_{i}, a_{j}\right) \in \psi \\
0, & \text { otherwise }
\end{array}\right.
$$

where $i=1,2, \ldots,|S|, j=1,2, \ldots,|A|$ and $\psi$ is a set of invalid state action pair.

The entire control procedure is described in Algorithm 1. The state representation in Fig. 2 yields a finite state space of dimension $2 \times 3$ making a total of 6 states. Also, there are a total of 13 possible actions to choose from. Hence, storing the $q$-table in memory is computationally feasible. Finally, the control methodology introduced in this section is general and can easily be extended for different use-cases.

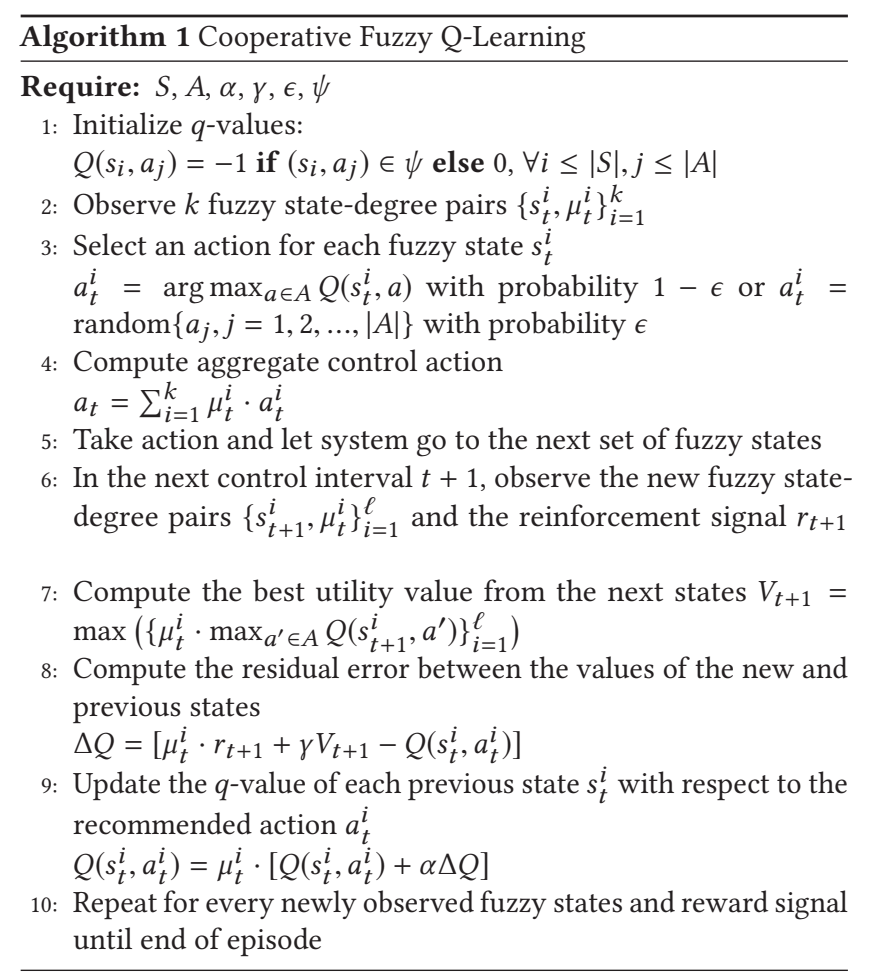

\section{EVALUATION}

In this section, we describe the experimental evaluation of the proposed adaptive performance control framework, CoFReL. The goal is to assess the efficacy and applicability of CoFReL for controlling the QoS of a real application benchmark running on a realistic virtualized testbed.

\subsection{Setup}

The experimental testbed is composed of an HP ProLiant Physical Machine (PM) equiped with a total of $32 \mathrm{CPU}$ cores and $56 \mathrm{~GB}$ of memory. The server runs a Xen hypervisor[3] to emulate a typical virtualized environment and to enable vertical auto-scaling. The service under test is the RUBiS ${ }^{4}$ web application benchmark, an eBay-like e-commerce application that provides selling, browsing and bidding functionalities. The service is deployed as a monolithic package in a single VM hosting an Apache 2.0 web server and MySQL 5.0 server. Since the focus is on compute capacity allocation, we configured the VM with $10 \mathrm{~GB}$ of memory to prevent unnecessary disk activity.

To generate realistic workload for the test, we emulated virtual web users concurrently interacting with the applications using an open source HTTP load generator, httpmon ${ }^{5}$. The tool dynamically generates realistic user behaviour by issuing HTTP GET or POST requests at varying intensities with exponential inter-arrival times. Requests are generated in a closed loop fashion according to three workload patterns as shown in Fig 3 a) STEP: abrupt increase or decrease in the number of concurrent users to emulate dynamic

\footnotetext{
${ }^{4}$ http://rubis.ow2.org/index.html

${ }^{5}$ https://github.com/cloud-control/httpmon
} 
changes in workload levels b) RAMP: continuous increase in the number of concurrent users to emulate traffic phenomena such as flash-crowds, c) TRI phase: a hybrid of the step and ramp patterns composed of three phases where the service experiences rapid increase in users, followed by a short period of constant number of users, and a downward ramp.

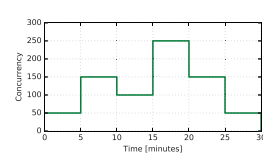

(a) STEP

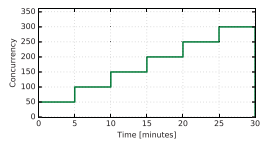

(b) RAMP

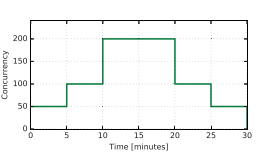

(c) TRI
Figure 3: Workload pattern

To monitor service performance, the Response Time, and resource usage, CPU utilization, we deployed a lightweight sensor to collect and send measurements to the controller running on the PM via UDP communication. The sampling rate was maintained at a 10s interval in all experiments. Capacity allocations and deallocation are actuated directly via the Xen hypervisor CPU hot-plugging $\mathrm{API}^{6}$.

\subsection{Performance Metrics}

The analysis of the proposed system is described in terms of 5 attributes namely

1) Achieved QoS: a summarized value of the observed performance (response time) aggregated over the entire experiment period.

2) Adjusted Integral of Absolute Error (AIAE): the Integral of Absolute Error aggregates the total errors (deviations) observed over an experiment given a control target. We adjust for controlling within a tolerance band by computing the deviation according to the following function:

$$
e_{t}= \begin{cases}R T_{t}-\tau^{+}, & R T_{t} \geq \tau^{+} \\ R T_{t}-\tau^{-}, & R T_{t} \leq \tau^{-} \\ R T_{t}-\tau, & \tau^{-} \leq R T_{t} \leq \tau^{+}\end{cases}
$$

where $\tau$ is the target service performance and $\tau^{-}$and $\tau^{+}$are the lower and upper limit of the tolerance region around the target respectively. The adjusted quantity is then computed as:

$$
A I A E=\sum\left|e_{t}\right|
$$

3) Allocation: the average resource capacity allocated to the service VM over an experiment in terms of the number of CPUs.

4) Efficiency: the ratio between the normalized average resource allocation and resource utilization

5) Adaptivity: the average number of monitoring epochs required to converge to the tolerance band after an overshoot or undershoot. We also report the average number of monitoring epochs required to converge after an abrupt increase (UP) and decrease (DOWN) in workload level, as well as the initial convergence rate (START).

\footnotetext{
${ }^{6}$ https://wiki.xen.org/wiki/Credit_Scheduler
}

\subsection{A RL-based Baseline Controller}

For the purpose of comparison we designed a baseline controller to determine if we are better off without the fuzzy state representation and parallel state exploration. Unlike the technique described in Section 4, the state detection mechanism is non-fuzzy and contains 3 discrete states. When the service performance is within the tolerance band, the system is said to be in a NORMAL state, VIOLATION if above the upper tolerance band, and LOW if below the lower tolerance band. The reward computation remains the same as in Section 4.2. The learning mechanism is based on the standard Q-learning algorithm involving only a single agent. A detail description of the basic Q-learning technique can be found in Sutton et al [22]. The action space and selection strategy are the same as described for CoFReL.

\subsection{Experiments and Results}

In this section, we describe and present the results of a number of experiments aimed at addressing some research questions. The objective is to examine how the proposed framework performs under different conditions.

All experiments were staged for a total length of 30 minutes, with workload intensity changing every 5 minutes. Unless otherwise stated, most of the experiments were run with a baseline configuration of target $\tau=1$ s, action selection strategy, $\epsilon=0.1$, learning rate, $\alpha=0.1$, and discount rate, $\gamma=0.5$. Since end-user experience is crucial, we prioritize SLA compliance over resource efficiency by setting $\omega=0.6$ in the utility function of Eq.(3). Also, for each experiment, the nominal compute capacity of the test VM was initialized to $1 \mathrm{CPU}$.

6.4.1 Time Series Analysis (RQ1). In order to characterize controller behaviour, we performed a total of nine experiments under varying load conditions and performance targets. Fig. 4 shows the time series plots of observed service performance, the allocated capacity as well as utilized capacity over time for the experiments. Each plot contains a control chart of service response time at the top and a line chart of allocated and utilized capacity at the bottom. The shaded region in the bottom chart represent unused capacity.

Clearly, the plots show that CoFReL adapt well with workload patterns while meeting performance requirements by scaling up and down in response to changes in workload levels. The plots also demonstrate the consistency of capacity allocations since roughly the same amount of capacity is allocated for a given workload level regardless of workload patterns. For the case of $1 \mathrm{~s}$ target, about 3 CPUs are allocated to handle 100 concurrent users in all three workload patterns. The controller also exhibit correct replay of learned scaling policies. For example, under the RAMP load, the amount of capacity allocated for 200 users remain the same for 250 and even 300 users since performance requirement continued to be met. Note that spikes and dips in the control charts are change points indicating sudden increase and decrease in service workload respectively.

6.4.2 Aggregate Analysis (RQ2). Here, we present aggregate analysis of controller behaviour for the experiments in Section 6.4.1. The results are summarized in Table 1 under 5 performance measures. 


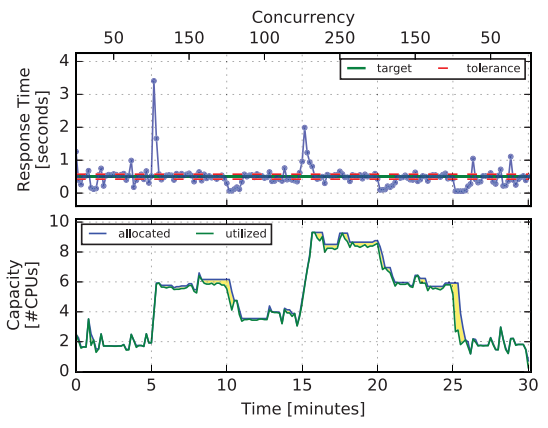

(a) STEP workload, target $=0.5 \mathrm{~s}$

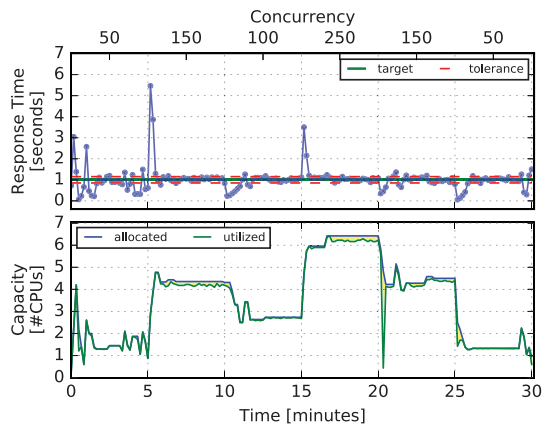

(d) STEP workload, target $=1 \mathrm{~s}$

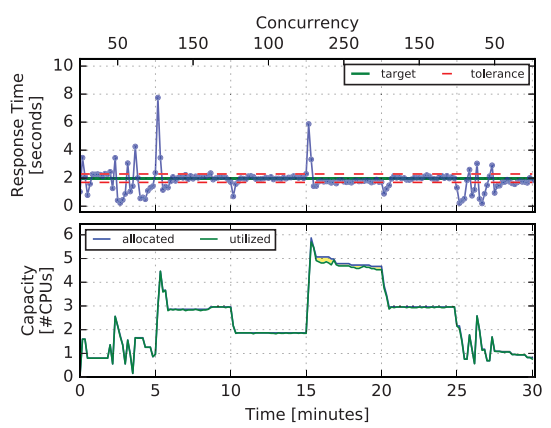

(g) STEP workload, target $=2 \mathrm{~s}$

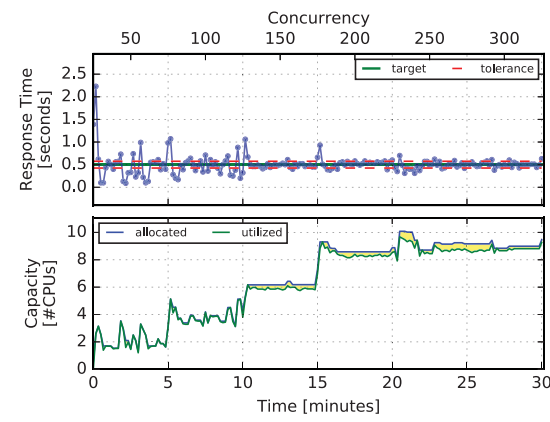

(b) RAMP workload, target $=0.5 \mathrm{~s}$
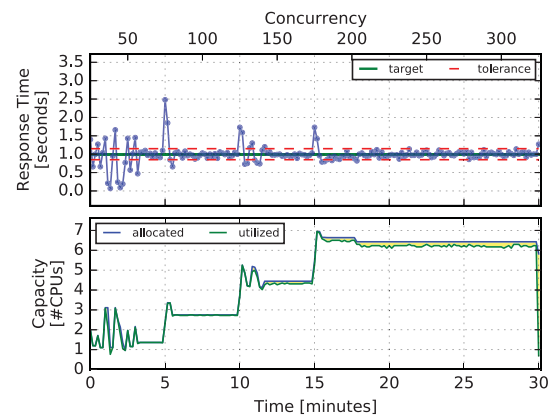

(e) RAMP workload, target $=1 \mathrm{~s}$
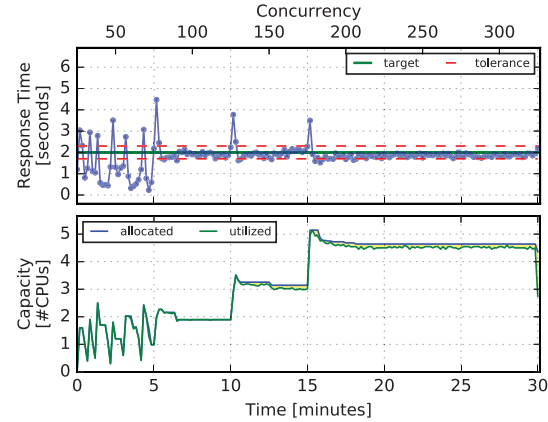

(h) RAMP workload, target $=2 \mathrm{~s}$

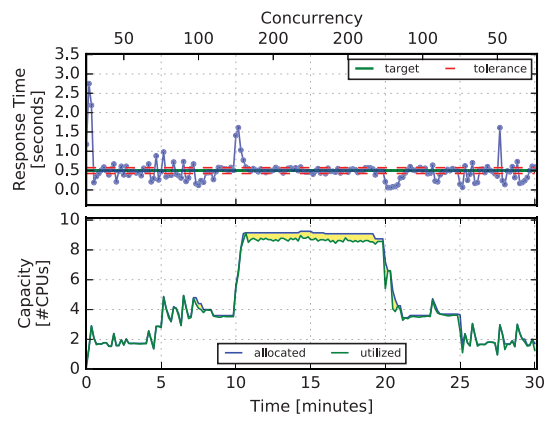

(c) TRI workload, target $=0.5 \mathrm{~s}$
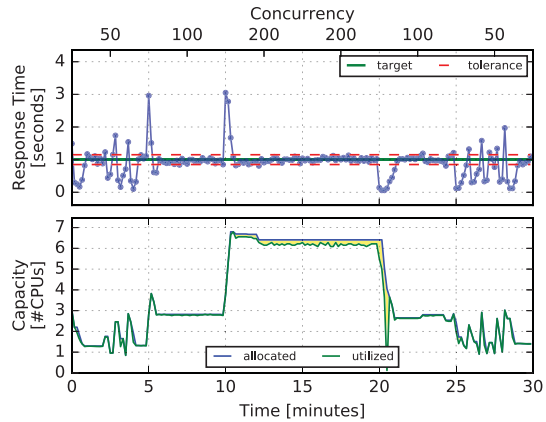

(f) TRI workload, target $=1 \mathrm{~s}$
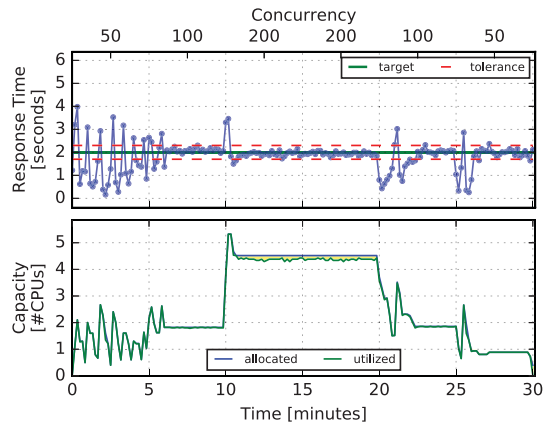

(i) TRI workload, target $=2 \mathrm{~s}$

Figure 4: Time series plots of service performance (average RT), allocated and utilized capacity under different QoS targets and workload patterns. Parameters: $(\epsilon=0.1, \alpha=0.1, \gamma=0.5)$

According to Table 1, CoFReL more easily meets performance requirement at a lower target of $0.5 \mathrm{~s}$ and $1 \mathrm{~s}$ than at higher target of $2 \mathrm{~s}$, but at a cost of lower resource efficiency. This observation remain consistent regardless of workload pattern. It is thus intuitive that it consumes more compute capacity at low targets (5.4 and 4 CPUs) than at the higher target (2.8 CPUs). The in ability to meet performance requirements at the $2 \mathrm{~s}$ targets can be explained by high performance variability (AIAE) and not under provisioning since $2 \%$ of allocated capacity is unused.
6.4.3 Adaptation (RQ3). The last column of Table 1 shows that CoFReL adapts faster in most cases when controlling for the $1 \mathrm{~s}$ target with an average of 6.7 epochs (i.e. $1.11 \mathrm{mins}$ at $10 \mathrm{~s}$ control interval). However, a break down of this value presented in Table 2 shows that CoFReL converge to the tolerance band within the first 11.3 epochs (1.88mins) on average. Furthermore, CoFReL takes 6.3 epochs (approximately $1 \mathrm{mins}$ ) to stabilize after an increase in workload level (scale up) and 12.6 epochs (2.1mins) after a decrease in workload level (scale down). Overall, the TRI load pattern has the fastest initial and scale-up adaptivity but also the slowest scaledown adaptivity. 
Table 1: Control performance of proposed controller under different targets and workloads

\begin{tabular}{|c|c|c|c|c|c|c|}
\hline \multirow[t]{2}{*}{ Target } & Workload & \multicolumn{2}{|c|}{$\begin{array}{l}\text { Achieved AIAE } \\
\text { QoS }\end{array}$} & \multirow{2}{*}{$\begin{array}{c}\text { Allocation } \\
4.8\end{array}$} & \multirow{2}{*}{$\begin{array}{c}\text { Efficiency } \\
96 \%\end{array}$} & \multirow{2}{*}{$\begin{array}{c}\text { Adaptivity } \\
7.9\end{array}$} \\
\hline & STEP & $0.5 \mathrm{~s}$ & 23 & & & \\
\hline \multirow{3}{*}{$0.5 s$} & RAMP & $0.5 \mathrm{~s}$ & 15 & 6.5 & $96 \%$ & 7.7 \\
\hline & TRI & $0.5 \mathrm{~s}$ & 22 & 5.0 & $96 \%$ & 7.9 \\
\hline & Mean & $0.5 \mathrm{~s}$ & 20 & 5.4 & $96 \%$ & 7.8 \\
\hline \multirow{4}{*}{$1 \mathrm{~s}$} & STEP & $1.01 \mathrm{~s}$ & 37 & 3.5 & $97 \%$ & 6.2 \\
\hline & RAMP & $0.99 \mathrm{~s}$ & 19 & 4.7 & $97 \%$ & 7.0 \\
\hline & TRI & $0.91 \mathrm{~s}$ & 36 & 3.7 & $97 \%$ & 7.0 \\
\hline & Mean & $0.97 \mathrm{~s}$ & 31 & 4.0 & $97 \%$ & 6.7 \\
\hline \multirow{4}{*}{$2 s$} & STEP & $1.88 \mathrm{~s}$ & 62 & 2.5 & $99 \%$ & 5.6 \\
\hline & RAMP & $1.81 \mathrm{~s}$ & 49 & 3.4 & $97 \%$ & 7.8 \\
\hline & TRI & $1.83 \mathrm{~s}$ & 54 & 2.6 & $98 \%$ & 16.3 \\
\hline & Mean & $1.84 \mathrm{~s}$ & 55 & 2.8 & $98 \%$ & 9.9 \\
\hline
\end{tabular}

Table 2: Convergence rates under different workload

\begin{tabular}{r|c|c|c|c}
\hline Workload & START & UP & DOWN & Mean \\
\hline STEP & 11.0 & 6.5 & 8.6 & 6.2 \\
RAMP & 16.0 & 8.5 & - & 7.0 \\
TRI & 7.0 & 4.0 & 16.5 & 7.0 \\
\hline Mean & 11.3 & 6.3 & 12.6 & 6.7 \\
\hline
\end{tabular}

6.4.4 Comparative Analysis (RQ4). We perform two sets of experiments which involve deploying CoFReL and the baseline controller of Section 6.3 independently to control a mean RT target of $1 \mathrm{~s}$ with the baseline configuration. Results in Table 3 show that using multiple agents to explore the fuzzy state space in parallel as implemented in CoFReL is more efficient than using only one single agent as in the case of the baseline. CoFReL meets the performance requirement with only $4 \mathrm{CPU}$ which is much lower than capacity allocated by the baseline controller. While the baseline controller never converges, CoFReL converges after 6.7 epochs on average.

Table 3: Performance comparison with a baseline controller

\begin{tabular}{|c|c|c|c|c|c|}
\hline & Achieved QoS & AIAE & Allocation & Efficiency & Adaptivity \\
\hline Baseline & $0.21 \mathrm{~s}$ & 127 & 23 & $50 \%$ & NA \\
\hline CoFReL & $0.97 \mathrm{~s}$ & 31 & 4.0 & $97 \%$ & 6.7 \\
\hline
\end{tabular}

6.4.5 Sensitivity Analysis (RQ5). Controller behaviour can be influenced in various ways such as by varying parameter settings. Also, disturbances from the service execution context, such as the influence of noisy tenants, can impact capacity allocations. Hence, we staged two sets of experiments to characterize these effects.

The first set of experiments involves systematically varying the value of each learning parameters while keeping others constant. Table 4 summarizes results from all experiments where the emboldened columns correspond to baseline parameter settings. For example, to obtain the results of varying the learning rate $(\alpha)$, we set the action selection strategy $(\epsilon)$ and discounting rate $(\gamma)$ to 0.1 and 0.5 respectively. The same methodology applied to the other two parameters. All experiments were performed with a target average RT of 1s under the STEP workload.

The baseline configuration offers the best control performance in terms of meeting the target (1.01s) by efficiently utilizing (97\%) the least amount of capacity allocations (3.5 CPUs) while offering the fastest adaptation rate (6.2 epochs) in most cases. Varying the learning rate does not seem to affect capacity allocations, resource efficiency, and controller adaptivity as much as the achieved QoS. The controller violates performance requirement and shows poor adaptivity when $\alpha=0.5$ with only $6 \%$ of allocated capacity unused. Increasing the probability of random action selection seems to overprovision in order to maintain performance stability except when $\epsilon=0.5$. The controller is only able to meet QoS requirement when there is a balance between future and immediate rewards (i.e. $\gamma=$ 0.5 ), otherwise it introduces oscillations that inhibits convergence to the tolerance region.

Lastly, we examine the effect of resource interference on capacity allocations by emulating a situation where the controlled service is colocated with another VM hosting a compute intensive service. A compute contention event is injected after the first 7 minutes of the experiment lasting for roughly 10 minutes by using the stress ${ }^{7}$ utility to spawn a set of workers spinning on sqrt (). The experiment is performed with an average RT target of 1s under the TRI workload while using the baseline learning parameter settings. Fig. 5 is the resulting time series plot of observed service $\mathrm{RT}$, capacity allocation and utilization. This plot is complementary
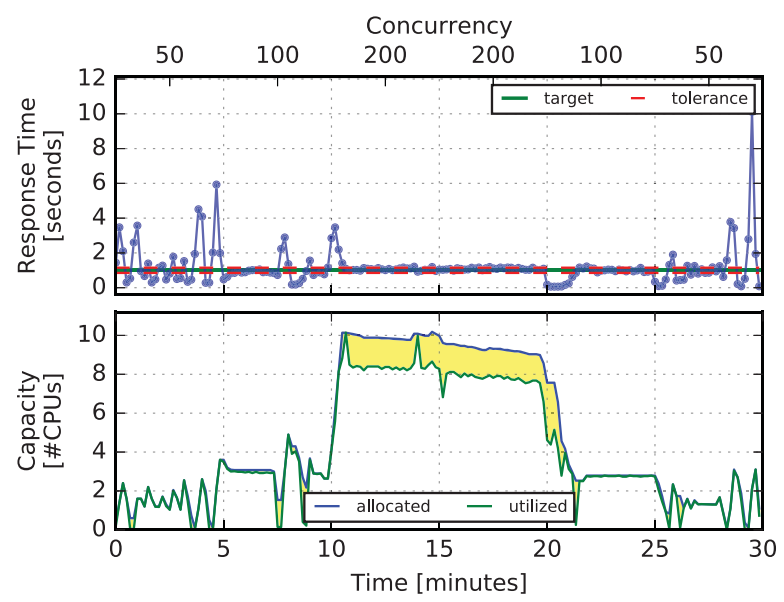

Figure 5: Impact of resource contention

to Fig. 4f, an instance of the same experiment where there is no contention. The impact of the event is immediate, causing undesirable variation at the start of the event. The immediate impact of the contention event is the observed anomalous variation in performance as well as capacity allocation and utilization around the 7th minute mark. The event also causes CoFReL to over-provision compute capacity in order to meet performance requirement. The obtained control performance is summarized in Table 5. Notice that

\footnotetext{
${ }^{7}$ https://people.seas.harvard.edu/ apw/stress/
} 
Table 4: Effects of varying learning parameters

\begin{tabular}{r|rrrrrrrrrrrrrrrr} 
& \multicolumn{1}{c}{ Action Selection Strategy $(\epsilon)$} & \multicolumn{4}{c}{ Learning Rate $(\alpha)$} & \multicolumn{3}{c}{ Discounting Rate $(\gamma)$} \\
\hline & $\mathbf{0 . 1}$ & 0.3 & 0.5 & 0.7 & 0.9 & $\mathbf{0 . 1}$ & 0.3 & 0.5 & 0.7 & 0.9 & 0.1 & 0.3 & $\mathbf{0 . 5}$ & 0.7 & 0.9 \\
\hline Achieved QoS & $\mathbf{1 . 0 1 s}$ & $0.91 \mathrm{~s}$ & $0.85 \mathrm{~s}$ & $0.93 \mathrm{~s}$ & $0.90 \mathrm{~s}$ & $\mathbf{1 . 0 1 s}$ & $1.10 \mathrm{~s}$ & $1.26 \mathrm{~s}$ & $1.04 \mathrm{~s}$ & $1.16 \mathrm{~s}$ & $1.11 \mathrm{~s}$ & $1.56 \mathrm{~s}$ & $\mathbf{1 . 0 1 s}$ & $1.34 \mathrm{~s}$ & $1.41 \mathrm{~s}$ \\
AIAE & $\mathbf{3 7}$ & 70.0 & 50.0 & 44.0 & 51.0 & $\mathbf{3 7}$ & 56.0 & 101.0 & 55.0 & 63.0 & 69.0 & 154.0 & $\mathbf{3 7}$ & 128.0 & 133.0 \\
Allocation & $\mathbf{3 . 5}$ & 8.0 & 6.2 & 6.1 & 6.1 & $\mathbf{3 . 5}$ & 3.5 & 3.5 & 3.6 & 3.5 & 3.8 & 3.7 & $\mathbf{3 . 5}$ & 3.8 & 3.8 \\
Efficiency & $\mathbf{9 7 \%}$ & $84 \%$ & $92 \%$ & $94 \%$ & $94 \%$ & $\mathbf{9 7 \%}$ & $96 \%$ & $94 \%$ & $95 \%$ & $94 \%$ & $94 \%$ & $93 \%$ & $\mathbf{9 7 \%}$ & $93 \%$ & $90 \%$ \\
Adaptivity & $\mathbf{6 . 2}$ & 8.3 & 10.4 & 13.4 & 27 & $\mathbf{6 . 2}$ & 6.7 & 8.3 & 5.3 & 3.9 & 10.6 & 10.8 & $\mathbf{6 . 2}$ & 8.1 & 11.2 \\
\hline
\end{tabular}

there is a high performance variation under the colocated scenario leading to performance violations. Aggregate resource efficiency is also hindered by the resource contention. Considering Fig. 4f, the unused capacity between the 10th and 20th time period has two indications; over-provisioning-because more capacity are allocated for the same workload level of 200 users as in Fig. 4f, approximately 6.5 CPUs compared to about 9 CPUs, and capacity deprivationsince the VM is only able to use about 8 CPUs of that perhaps due to stolen CPU cycles by the antagonist tenant.

Table 5: Control performance under difference execution contexts

\begin{tabular}{lc|c|c|c|c}
\hline Context & Achieved QoS & AIAE & Allocation & Efficiency & Adaptivity \\
\hline Isolated & $0.91 \mathrm{~s}$ & 36 & 3.7 & $97 \%$ & 7 \\
Colocated & $1.17 \mathrm{~s}$ & 76 & 4.7 & $86 \%$ & 11 \\
\hline
\end{tabular}

\section{RELATED WORK}

There is a large body of research contributions addressing the autoscaling of distributed and/or virtualized services [17, 19]. In this section, we classify the most relevant works into three categories namely ruled-based, model-driven and model-free approaches.

Rule-based approaches employ the use of threshold-based rules to specify autoscaling policies that determine when and how much to scale. For instance, Chieu et al [4] provision additional VM instances when the scaling indicators (e.g. CPU utilization) of all indicators are above a certain threshold and turn off idle instances otherwise. A similar approach is employed in [10] to scale up the number of VM instances when CPU utilization is high. Hasan et al [9] developed sophisticated threshold strategies based on correlation between resources to achieve integrated autoscaling. More sophisticated rule-based controllers such as RobustT2Scale [11] rely on fuzzy rules for horizontal scaling. Such fuzzy rules have also been applied in [6] to coordinate the scaling decision of both a CPU and memory controllers. Rules, however, rely on detail knowledge of services or workloads while associated thresholds may easily become obsoletes. Though the design of the proposed approach involves the use of fuzzy rules, this is only used for state recognition and not for learning optimal control actions.

Model-based approaches rely on different types of models of services and workloads to allocate resources correctly. Such models typically capture the propagation of transactions across distributed components or estimate the relationship between workload and performance or resource consumption. Queueing theory is very popular in the design of controllers. The horizontal scaling mechanism in [23] estimates capacity demands of multi-tier web applications using queueing networks with a different model for each time-scale. A queueing based analytical model is presented in [1] for horizontal scaling but evaluated in a simulated environment. To meet performance requirement, Lakew et al [15] introduced a fine-grained vertical scaling technique based on the inverse relationship between service latency and allocated capacity. A layered approach for vertical scaling is presented in [21] which uses both a queueing-theoretic model and a feedback controller. The goal is to meet SLA requirements via fine-grained vertical autoscaling. Ap$\mathrm{pRM}$, a hierarchical control mechanism is introduced in [18]. The technique used a feedback controller to adjusts capacity allocations for each application and a cluster-wide controller to account for potential contentions within a pool of application. Wahajat et al [25] presents MLScale, a technique for preventing SLA violations via blackbox horizontal scaling. MLScale builds a performance model capturing the relationship between SLA violation and low level resource metrics using neural networks and statistical regression. Similarly, Rameshan et al [20] used a binary SVM classifier to capture the relationship between resource consumption and state of SLO compliance. The main objective is to investigate impact of resource interference on controller behaviour and finding a means to factor in such interference. Unlike most of the aforementioned techniques, the proposed technique is easily amenable to varying workload, objectives and scaling scenario (e.g. tail or mean targets, scale-up or scale-down) since it does not require precise service models. Model-free approaches typically apply reinforcement learning (RL) algorithms for autonomously learning optimal autoscaling policies. Dutreilh et al [5] employed a single decision agent to learn the number of VMs to be allocated via Q-learning. However, the state representation requires knowledge of service workloads. A RL-based mechanism is introduced in [16] to achieve distributed resource provisioning and placement in cloud environments. Distribution is achieved via explicit knowledge sharing where controllers publish their Q-values to peers in the network. The design of FQL4KE [12] is similar to our proposed mechanism in that the goal is towards achieving QoS control using fuzzy Qlearning. However, the difference is that while FQL4KE focused on horizontal scaling, CoFReL instead deals with the vertical scaling problem. In addition, unlike CoFReL, FQL4KE's state representation requires knowledge of service workloads while the system can only assume a single state. Finally, FQL4KE involves a single learning agent to adapt its fixed set of control rules. Kontarinis et al [13] 
formulated horizontal scaling as an MDP problem and applied Qlearning to learn optimal scaling policies. However, it is not suitable under dynamic workloads as states are formulated as a function of workload and capacity allocation. The closest technique to the proposed mechanism is Auto-scale [24], an RL-based technique for adaptive QoS control via vertical scaling using Neuro-Fuzzy function approximations. However, the focus is only on meeting performance target without regards for efficiency. Besides relying on workload behaviour in its state representation, the convergence rate of the controller was not investigated. CoFReL is designed to strike a balance between meeting desired performance SLA and resource efficiency without explicit service or workload model. The technique strives for fast online adaptation by using multiple RL agents to explore the state space in parallel. Also, the fuzzy state formulation not only aids adaptation, it also enhances the effect of actions via fine-grained actuations.

\section{CONCLUSION}

Dynamic resource provisioning towards meeting end-user performance SLA while ensuring efficient utilization of allocated capacity remains a challenge in virtualized environments. While most commercial solutions rely on thresholding rules that adapt poorly with workloads researchers have investigated sophisticated models that are often too cumbersome to adapt to different scaling scenarios and sometimes require knowledge of service or workload characteristics. In this paper, we present a generic approach for adaptive performance control in virtualized environments using cooperative fuzzy reinforcement learning. In particular, we focus on minimizing the long training and adaptation time that inhibit applicability of $\mathrm{RL}$ techniques by formulating the state space as a two-dimensional fuzzy space and deploying multiple goal-seeking agents to explore the state space while actively sharing their experience. Using a real experimental testbed and application benchmark, we evaluated the efficacy of proposed mechanism in meeting performance SLA targets under varying conditions as well as assessing the impact of resource interference on its behaviour. Future work will focus on comparison with a variety of model-based techniques,integrate both vertical and horizontal scaling, and addressing interferenceaware autoscaling.

\section{ACKNOWLEDGMENT}

This work is supported by the Swedish Research Council (VR) under contract C0590801 for the Cloud Control project and the Swedish Strategic Research Program (eSSENCE).

\section{REFERENCES}

[1] Ahmed Ali-Eldin, Johan Tordsson, and Erik Elmroth. 2012. An Adaptive Hybrid Elasticity Controller for Cloud Infrastructures. In Network Operations and Management Symposium (NOMS), 2012 IEEE. IEEE, 204-212.

[2] Ying Bai and Dali Wang. 2006. Fundamentals of Fuzzy Logic Control-Fuzzy Sets, Fuzzy Rules and Defuzzifications. In Advanced Fuzzy Logic Technologies in Industrial Applications. Springer, 17-36.

[3] Paul Barham, Boris Dragovic, Keir Fraser, Steven Hand, Tim Harris, Alex Ho, Rolf Neugebauer, Ian Pratt, and Andrew Warfield. 2003. Xen and the Art of Virtualization. ACM SIGOPS Operating Systems Review 37, 5 (2003), 164-177.

[4] Trieu C Chieu, Ajay Mohindra, and Alexei A Karve. 2011. Scalability and Performance of Web Applications in a Compute Cloud. In 8th International Conference on e-Business Engineering (ICEBE). IEEE, 317-323.

[5] Xavier Dutreilh, Sergey Kirgizov, Olga Melekhova, Jacques Malenfant, Nicolas Rivierre, and Isis Truck. 2011. Using Reinforcement Learning for Autonomic
Resource Allocation in Clouds: Towards a Fully Automated Workflow. In ICAS 2011, The Seventh International Conference on Autonomic and Autonomous Systems. 67-74.

[6] Soodeh Farokhi, Ewnetu Bayuh Lakew, Cristian Klein, Ivona Brandic, and Erik Elmroth. 2015. Coordinating CPU and Memory Elasticity Controllers to Meet Service Response Time Constraints. In International Conference on Cloud and Autonomic Computing (ICCAC). IEEE, 69-80.

[7] Anshul Gandhi, Parijat Dube, Alexei Karve, Andrzej Kochut, and Li Zhang. 2014 Adaptive, Model-driven Autoscaling for Cloud Applications.. In ICAC, Vol. 14 57-64.

[8] Anshul Gandhi, Mor Harchol-Balter, Ram Raghunathan, and Michael A Kozuch 2012. Autoscale: Dynamic, Robust Capacity Management for Multi-tier Data Centers. ACM Transactions on Computer Systems (TOCS) 30, 4 (2012), 14.

[9] Masum Z Hasan, Edgar Magana, Alexander Clemm, Lew Tucker, and Sree Lakshmi D Gudreddi. 2012. Integrated and Autonomic Cloud Resource Scaling. In Network Operations and Management Symposium (NOMS), 2012. IEEE, 1327-1334.

[10] Waheed Iqbal, Matthew N Dailey, David Carrera, and Paul Janecek. 2011. Adaptive Resource Provisioning for Read Intensive Multi-tier Applications in the Cloud Future Generation Computer Systems 27, 6 (2011), 871-879.

[11] Pooyan Jamshidi, Aakash Ahmad, and Claus Pahl. 2014. Autonomic Resource Provisioning for Cloud-based Software. In Proceedings of the 9th International Symposium on Software Engineering for Adaptive and Self-Managing Systems. ACM, 95-104.

[12] Pooyan Jamshidi, Amir Sharifloo, Claus Pahl, Hamid Arabnejad, Andreas Metzger and Giovani Estrada. 2016. Fuzzy Self-learning Controllers for Elasticity Management in Dynamic Cloud Architectures. In 12th International ACM SIGSOFT Conference on Quality of Software Architectures (QoSA), 2016. IEEE, 70-79.

[13] Alexandros Kontarinis, Verena Kantere, and Nectarios Koziris. 2016. Cloud Resource Allocation from the User Perspective: A Bare-Bones Reinforcement Learning Approach. In International Conference on Web Information Systems Engineering. Springer, 457-469.

[14] L. I. Kuncheva. 2008. Fuzzy Classifiers. Scholarpedia 3, 1 (2008), 2925. Revision 133818.

[15] Ewnetu Bayuh Lakew, Cristian Klein, Francisco Hernandez-Rodriguez, and Erik Elmroth. 2014. Towards Faster Response Time Models for Vertical Elasticity In Proceedings of the 2014 IEEE/ACM 7th International Conference on Utility and Cloud Computing. IEEE Computer Society, 560-565.

[16] Han Li and Srikumar Venugopal. 2011. Using Reinforcement Learning for Controlling an Elastic Web Application Hosting Platform. In Proceedings of the 8th ACM international Conference on Autonomic Computing. ACM, 205-208.

[17] Tania Lorido-Botran, Jose Miguel-Alonso, and Jose Antonio Lozano. 2014. A Review of Auto-scaling Techniques for Elastic Applications in Cloud Environments. 7. Grid Comput. 12, 4 (2014), 559-592.

[18] Lei Lu, Xiaoyun Zhu, Rean Griffith, Pradeep Padala, Aashish Parikh, Parth Shah, and Evgenia Smirni. 2014. Application-driven Dynamic Vertical Scaling of Virtual Machines in Resource Pools. In Network Operations and Management Symposium (NOMS), 2014 IEEE. IEEE, 1-9.

[19] Chenhao Qu, Rodrigo N Calheiros, and Rajkumar Buyya. 2016. Auto-scaling Web Applications in Clouds: a Taxonomy and Survey. arXiv preprint arXiv:1609.09224 (2016).

[20] Navaneeth Rameshan, Ying Liu, Leandro Navarro, and Vladimir Vlassov. 2016. Elastic Scaling in the Cloud: A Multi-Tenant Perspective. In IEEE 36th International Conference on Distributed Computing Systems Workshops (ICDCSW), 2016. IEEE, $25-30$.

[21] Simon Spinner, Samuel Kounev, Xiaoyun Zhu, Lei Lu, Mustafa Uysal, Anne Holler and Rean Griffith. 2014. Runtime Vertical Scaling of Virtualized Applications via Online Model Estimation. In IEEE Eighth International Conference on Self-Adaptive and Self-Organizing Systems (SASO). IEEE, 157-166.

[22] Richard S Sutton and Andrew G Barto. 1998. Reinforcement learning: An Introduction. Vol. 1. MIT press Cambridge.

[23] Bhuvan Urgaonkar, Prashant Shenoy, Abhishek Chandra, Pawan Goyal, and Timothy Wood. 2008. Agile Dynamic Provisioning of Multi-tier Internet Applications. ACM Transactions on Autonomous and Adaptive Systems (TAAS) 3, 1 (2008), 1.

[24] T Veni and S Mary Saira Bhanu. 2016. Auto-scale: Automatic Scaling of Virtualised Resources Using Neuro-Fuzzy Reinforcement Learning Approach. International fournal of Big Data Intelligence 3, 3 (2016), 145-153.

[25] Muhammad Wajahat, Anshul Gandhi, Alexei Karve, and Andrzej Kochut. 2016. Using Machine Learning for Black-box Autoscaling. In Seventh International Conference on Green and Sustainable Computing Conference (IGSC0). IEEE, 1-8. 